〔Med. Entomol. Zool. Vol. 57 No. 3 p. 219-227 2006〕

\title{
A new species of Simulium (Simulium) (Diptera: Simuliidae) from Taiwan
}

\author{
Hiroyuki TAKAOKA ${ }^{1)}$ and Yao-Te HuAng ${ }^{2)}$ \\ ${ }^{1)}$ Department of Infectious Disease Control, Faculty of Medicine, Oita University, \\ Hasama, Yufu, Oita, 879-5593 Japan \\ 2) Department of Environmental Entomology, Rakuno Gakuen University, \\ Bunkyodaimidori-machi, Ebetsu, Hokkaido, 068-8501 Japan
}

(Received: 27 March 2006; Accepted: 22 May 2006)

\begin{abstract}
Simulium (Simulium) chungi sp. nov. is described based on adult, pupal and larval specimens collected from Taiwan. This is assigned to the griseifrons species-group, and is very similar to S. (S.) taipokauense Takaoka, Davies and Dudgeon, described from males collected from Hong Kong but there are differences in several morphological features including the relative length of the first flagellar segment of the antenna to the second one, the shape and size of the sensory vesicle, and the color of the midlegs.
\end{abstract}

Key words: black fly, Simulium, Simuliidae, Taiwan, new species

The fauna of the family Simuliidae in Taiwan has been sporadically studied, and only 23 species have been so far reported (Chung, 1986; Shiraki, 1935; Takaoka, 1979). All these species are assigned to the genus Simulium Latreille s. 1., and are, within it, also classified to six subgenera, Eusimulium Roubaud (1 species), Gomphostilbia Enderlein (2 species), Montisimulium Rubtsov (1 species), Nevermannia Enderlein (5 species), Simulium Latreille s. str. (12 species) and Wallacellum Takaoka (1 species) (Takaoka, 1979, 2003). During recent surveys on aquatic stages of black flies in Taiwan, we collected one new species of the subgenus Simulium s. str., which is described in this paper.

The terms for morphological features used here follow those of Takaoka (2003). Holotype and paratype specimens of the new species are deposited at the Department of Infectious Disease Control, Faculty of Medicine, Oita University, Oita, Japan.

\section{Simulium (Simulium) chungi sp. nov.}

DESCRIPTION. Female. Body length 2.2$2.4 \mathrm{~mm}$. Head. Narrower than thorax. Frons brownish-black, shiny, with several dark stout hairs along lateral and lower margins; frontal ratio $1.28-1.29: 1.00: 1.04-1.26$; frons-head ratio $1.00: 3.62-3.75$. Fronto-ocular area (Fig. 1 A) well developed, triangular, directed laterally and slightly upward. Clypeus brownish-black, thickly white pruinose, slightly shiny at certain angle of light, moderately covered with dark stout hairs intermixed with a few pale slender hairs except upper $2 / 3$ longitudinally bare medially. Labrum 0.65 times as long as clypeus. Antenna composed of $2+9$ segments, with color variable in specimens: in some females, antenna medium brown to brownishblack except scape, pedicel, and base of 1st flagellar segment yellow when viewed posteriorly, and medium brown to brownish-black except scape, pedicel, 1st and 2nd (sometimes also 3rd) flagellar segments yellow when viewed anteriorly; in other females, antenna almost yellow except apex somewhat dark. Maxillary palp light to dark brown, composed of 5 segments, with proportional lengths of 3 rd, 4 th, and 5 th segments $1.0: 1.1: 2.6-2.8$; 3rd 


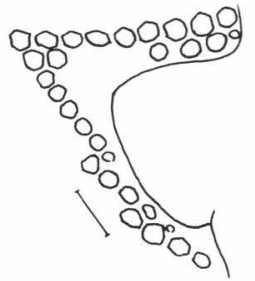

A

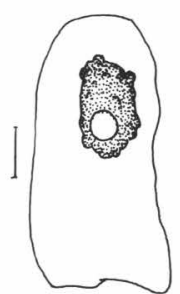

B

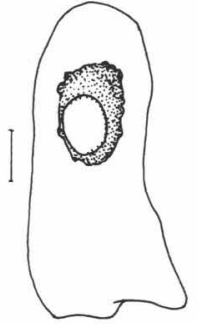

C

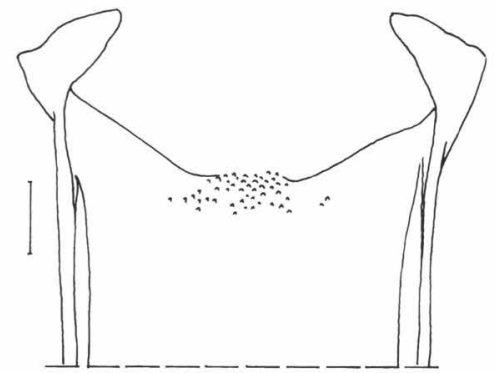

D
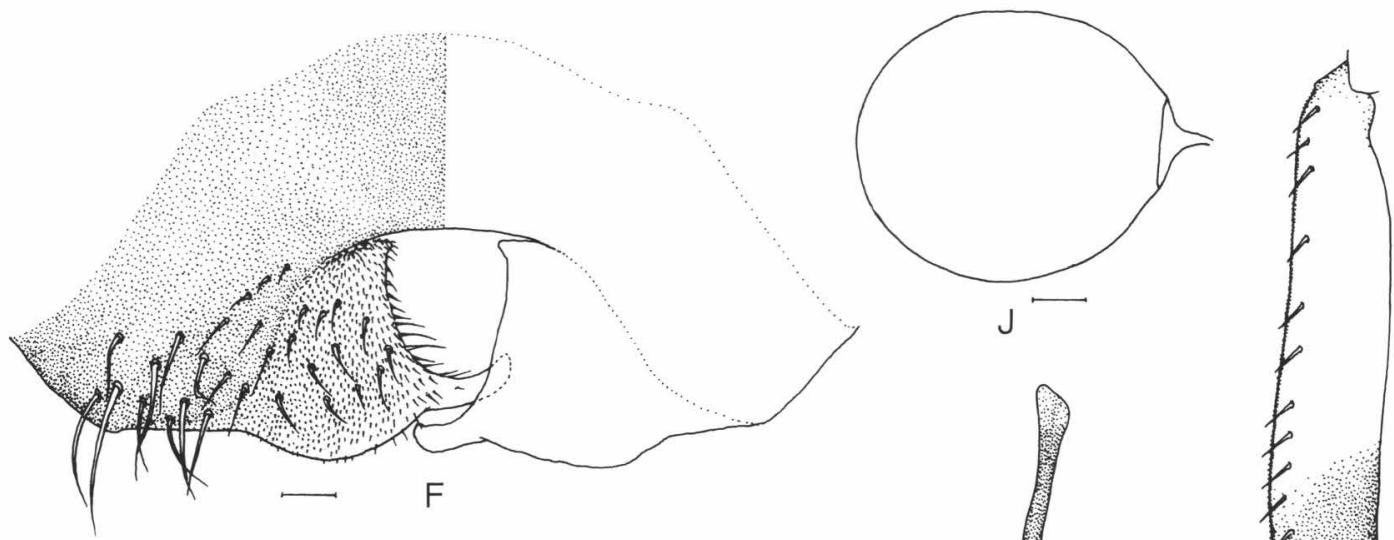

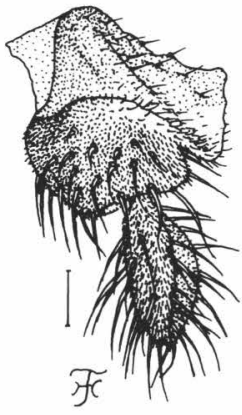

$\mathrm{H}$

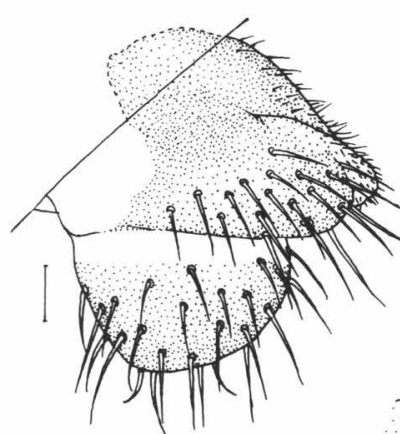

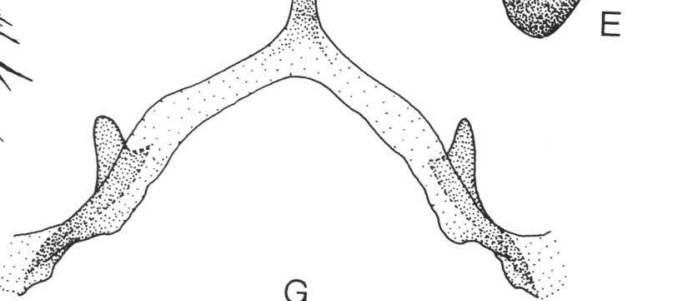

Fig. 1. Adult female of Simulium (Simulium) chungi sp. nov. A, Fronto-ocular area; B and C, 3rd segments of maxillary palp showing sensory vesicle (right side, front view); D, cibarium; E, basitarsus and 2nd tarsal segment of hind leg (left side, outer view); F, 8th sternite and ovipositor valves in situ (ventral view); G, genital fork (ventral view); H and I, paraprocts and cerci (right side; H, ventral view; I, outer view); J, spermatheca. Scale bars. $0.1 \mathrm{~mm}$ for E; $0.03 \mathrm{~mm}$ for A; $0.02 \mathrm{~mm}$ for B-D and F-J.

segment (Fig. 1B, C) of normal size, with oblong sensory vesicle of moderate size $(0.35-0.38$ times as long as 3rd segment) having medium to very large opening apically. Maxillary lacinia with 10-13 inner and 11-13 outer teeth. Mandible with 21 or 22 inner and 12 outer teeth. Cibarium (Fig. 1D) with many minute processes medially near posterior margin. Thorax. Scutum black, shiny, densely covered with whitish-yellow recumbent short hairs interspersed with several dark long upright hairs on prescutellar area; when illuminated in front and viewed dorsally, scutum thickly white pruinose with 5 non-pruinose longitudinal vittae, 
of which 1 median vitta is of moderate width, 2 submedian and 2 sublateral vittae rather wide though sublateral vittae somewhat wider than submedian ones, all vittae united with broad non-pruinose transverse band on prescutellar area; when illuminated from behind, scutum having reversed color pattern. Scutellum medium to dark brown, covered with dark long upright hairs as well as whitish-yellow short hairs. Postnotum dark brown to brownishblack, shiny, grey pruinose, bare. Pleural membrane bare. Katepisternum longer than deep, bare. Legs. Foreleg: coxa and trochanter whitish-yellow; femur yellow with apical cap light yellowish-brown; tibia white except apical cap brownish-black, with whitish sheen on outer surface when illuminated at certain angle of light; tarsus brownish-black, with moderate dorsal hair crest; basitarsus moderately dilated, 5.5 times as long as its greatest width. Midleg: coxa brownish-black; trochanter and femur yellow; tibia white to yellowish-white except apical tip light brown, with white sheen on posterior surface when illuminated at certain angle of light; tarsus brownish-black except basal $3 / 5$ of basitarsus yellowish-white. Hind leg: coxa dark brown; trochanter yellow; femur yellow with apical cap dark brown; tibia yellowish-white with apical $1 / 3$ brownish-black, with white sheen on posterior surface when illuminated at certain angle of light; tarsus blackish-brown except basal $3 / 5$ of basitarsus, and basal $1 / 2$ of 2 nd segment yellowish-white; basitarsus (Fig. 1E) nearly parallel-sided, 5.8 times as long as wide, and 0.79 and 0.67 times as wide as greatest widths of hind tibia and femur, respectively; calcipala (Fig. 1E) moderately developed, slightly shorter than its basal width, not reaching level of pedisulcus and 0.38 times as wide as greatest width of basitarsus; pedisulcus (Fig. 1E) well developed at basal $2 / 5$ of 2 nd tarsal segment. All tarsal claws simple. Wing. Length 2.3-2.4 mm. Costa with dark spinules and hairs; subcosta haired except apical $1 / 4$ to $1 / 3$ bare; basal section of radial vein haired except basal $1 / 6$ to $1 / 5$ bare; $R_{1}$ with dark spinules and hairs; $\mathrm{R}_{2}$ with hairs; hair tuft on stem vein dark brown; basal cell absent. Abdomen. Basal scale dark brown to brownish-black, with fringe of pale hairs. Dorsal surface of abdomen dark brown to brownish-black except basal $1 / 2$ or a little less of 2 nd segment yellowish-white, with short dark hairs; tergite 2 shiny, white iridescent when illuminated at certain angle of light, and tergites 6-8 shiny. Ventral surface of segment 7 with pair of large weakly sclerotized sternal plates medially. Genitalia. Sternite 8 (Fig. 1F) deeply concave posteriorly, with 20-22 dark short to long hairs on each lateral surface. Ovipositor valves (Fig. 1F) nearly triangular, rounded ventrally, with narrow almost bare tip posteromedially, membranous, moderately covered with microsetae interspersed with $13-$ 15 pale short hairs; inner margins deeply concave, widely separated from each other but their tips crossing poteromedially. Genital fork (Fig. 1G) of inverted-Y form, with narrow wellsclerotized stem; arms of moderate width, each with distinct projection directed anterodorsally. Paraproct in ventral view (Fig. 1H) with anterior $1 / 2$ strongly sclerotized and having deep bare depression on anterolateral surface and 12-15 sensilla on anteromedial surface; paraproct with about 20 short to mediumlong hairs as well as numerous microsetae on ventral and lateral surfaces of posterior $1 / 2$; paraproct in lateral view (Fig. 1I) much protruding ventrally beyond vental margin of cercus, tapered from middle toward ventral tip. Cercus (Fig. 1H, I) short, about half as long as wide, with numerous stout hairs and rounded posteriorly when viewed laterally. Spermatheca (Fig. 1J) nearly ovoid, well sclerotized except small portion of juncture to duct unsclerotized, with no distinct reticulate pattern on its surface, and with internal setae; accessory ducts subequal in diameter to each other, and slightly thicker than major duct.

Male. Body length 2.5-2.6 mm. Head. Width subequal to that of thorax. Upper eye consisting of large facets in 19 or 20 vertical columns and in 19 or 20 horizontal rows. Clypeus black, thickly white pruinose, covered with dark brown hairs along and near lateral margins but bare on most of central portion. Antenna composed of $2+9$ segments, dark brown except scape and pedicel dark yellow to light brown, and basal $1 / 2$ or a little less of 1 st flagellar segment yellow; 1st flagellar segment elongate, 1.5-1.6 times as long as 2nd one. Maxillary palp dark brown, composed of 5 segments, with proportional lengths of $3 \mathrm{rd}$, 4 th, and 5 th segments $1.0: 1.3-1.4: 2.7-3.0$; 3rd segment (Fig. 2A, B) of normal size; sensory vesicle small (0.24-0.28 times as long as 3rd segment), ellipsoidal or oblong, and with medium-sized opening near apex. Thorax. Scutum black, 


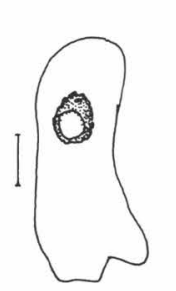

A

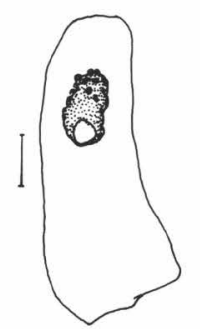

$\mathrm{B}$

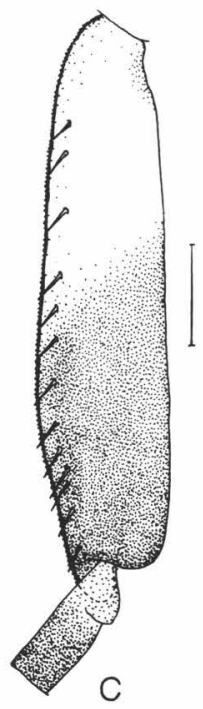

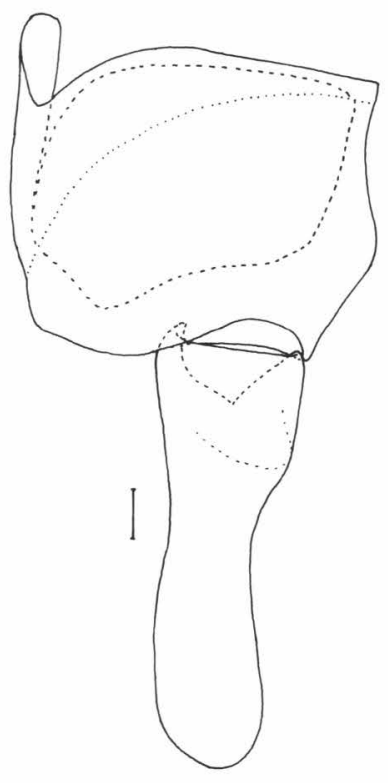

E
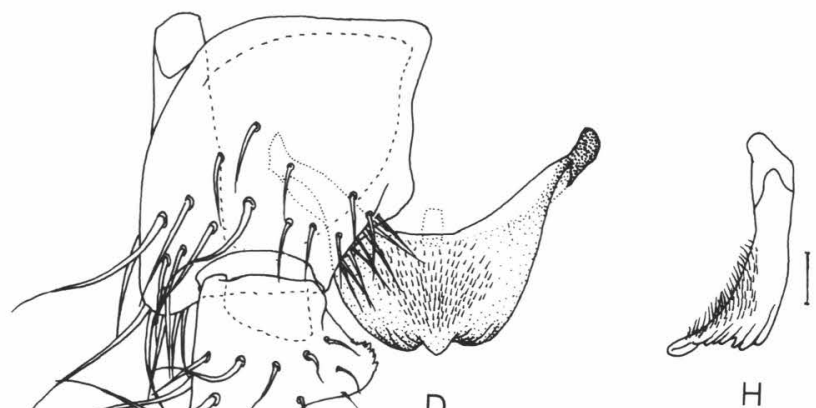

$\mathrm{H}$
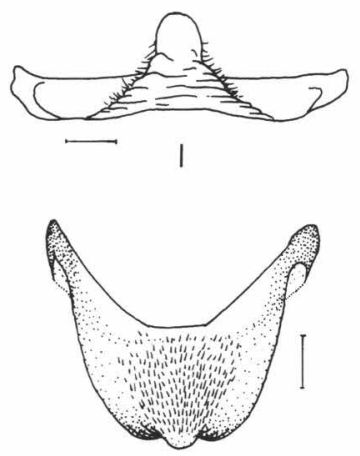

G

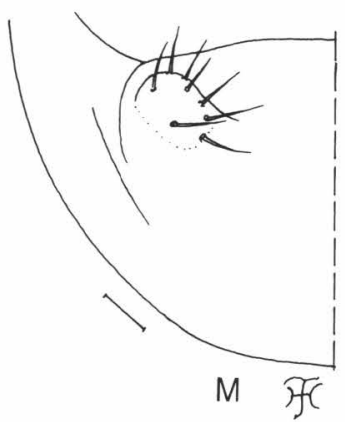

Fig. 2. Adult male of Simulium (Simulium) chungi sp. nov. A and B, 3rd segments of maxillary palp with sensory vesicle (right side, front view); C, basitarsus and 2nd tarsal segment of hind leg (left side, outer view); D, right coxite, right style and ventral plate in situ (ventral view); E, coxite and style (right side, ventrolateral view); F, right style with basal protuberance (outer view); G-I, ventral plates ( $G$, ventral view; H, lateral view; I, end view); J, median sclerite (end view); K, paramere (right side, end view); $\mathrm{L}$ and $\mathrm{M}, 10$ th abdominal segments with cercus on right side (L, outer view; $\mathrm{M}$, end view). Scale bars. $0.1 \mathrm{~mm}$ for $\mathrm{C} ; 0.02 \mathrm{~mm}$ for $\mathrm{A}, \mathrm{B}$ and $\mathrm{D}-\mathrm{M}$.

with white pruinose pattern, i.e., anterior pair of rectangular spots on shoulders extending posteriorly along lateral margins and connected to large transverse posterior spot covering prescutellar area; these pruinose areas silvery iridescent when illuminated at certain angle of light; scutum uniformly and densely covered with golden-yellow recumbent short hairs interspersed with dark brown long upright hairs on prescutellar area. Scutellum black, shiny, white pruinose, with several dark long upright hairs as well as golden-yellow short hairs. 
Postnotum black, shiny when illuminated at certain angle of light. Pleural membrane and katepisternum as in female. Legs. Foreleg: coxa yellowish-white; trochanter and femur light brown though most of inner surface of femur yellow; tibia medium brown to blackishbrown, and with large white sheen when illuminated at certain angle of light; tarsus black, with moderate dorsal hair crest; basitarsus moderately dilated, 6.1 times as long as its greatest width. Midleg: coxa brownish-black; trochanter medium brown except basal 1/2 yellow; femur yellow to somewhat dark yellow with apical cap light brown; tibia yellow except apical cap and apical $1 / 2$ or more of posterior surface slightly to moderately darkened; tarsus brownish-black to black except basal $3 / 5$ of basitarsus yellow. Hind leg: coxa brownish-black; trochanter yellow to dark yellow; femur dark yellow to light brown with apical cap dark brown though inner and anterior surfaces of femur widely yellow; tibia brownish-black except basal tip white; tarsus brownish-black except basal $2 / 5$ or a little more of basitarsus yellow and basal $1 / 3$ of 2 nd tarsal segment medium brown; basitarsus (Fig. 2C) much enlarged, gradually widened from base to apical $2 / 3$, then gradually narrowed to apex, 4.1 times as long as its greatest width, and 0.9 times as wide as greatest width of hind tibia which is as wide as that of hind femur; calcipala small, slightly shorter than its basal width; pedisulcus well marked. Wing. Length $2.1-2.3 \mathrm{~mm}$. Other characters as those of female except subcosta and basal portion of radial vein entirely bare. Abdomen. Basal scale black, with fringe of dark long hairs. Dorsal surface of abdomen black, with dark short hairs; segments 2 and 5-7 each with pair of silvery iridescent spots dorsolaterally, those on segment 2 connected broadly to each other in middle. Genitalia. Coxite in ventral and ventrolateral views (Fig. 2D, E) nearly quadrate. Style in ventral view (Fig. 2D) elongate, with outer margin nearly straight and inner margin moderately concave, with basal protuberance directed mediodorsally, and without apical spine; style in ventrolateral view (Fig. 2 E) gradually narrowed from base to middle, then slightly widened toward apex, 2.9 times as long as its greatest width at base and 5.2 times as long as its narrowest width at middle; style in lateral view (Fig. 2F) flattened ventrodorsally, with long basal protuberance pointed mediodorsally and bearing many coneshaped spines on its tip and anterior surface of apical $1 / 2$. Ventral plate in ventral view (Fig. 2 D, G) with saddle-shaped body, which is gradually narrowed posteriorly, irregularly furrowed on posterior surface, and has many minute setae on ventral surface except each lateral area bare; basal arms short, stout, diverged from each other at angle of 90 degrees or more; ventral plate in lateral view (Fig. 2H) with ventral margin gently curved ventrally and appearing to have dentate posterior margin; ventral plate in end view (Fig. 2I) with body equilaterally triangular in shape, with small rounded apex, and with several transverse furrows on posterior surface. Median sclerite (Fig. 2J) wide, thin, plate-like. Paramere (Fig. 2K) with many parameral hooks. Aedeagal membrane moderately covered with minute setae; dorsal plate weakly sclerotized except median area widely unsclerotized. Ventral surface of 10 th abdominal segment without any distinct hairs. Cercus (Fig. 2L, M) rounded, with 7-9 distinct hairs.

Pupa. Body length $2.5-3.0 \mathrm{~mm}$. Head. Integument including antennal sheaths dark yellow, densely and regularly covered with small round tubercles; frons with 2 simple medium-long trichomes (Fig. 3A) lying close together on each side; face with 1 simple long trichome (Fig. 3B) on each side. Thorax. Integument dark yellow to yellowish-brown, densely and regularly covered with small round and conical tubercles; thorax on each side with 3 very long stout trichomes each simple or split into $2-4$ branches (Fig. 3C) mediodorsally, 2 simple stout trichomes (1 very long, 1 medium-long) (Fig. 3D) anterolaterally, 1 simple medium-long stout trichome (Fig. 3E) posterolaterally, and 2 simple long stout trichomes and 1 simple medium-long slender trichome (Fig. $3 \mathrm{~F}$ ) ventrolaterally. Gill (Fig. 3G) with 6 short slender thread-like filaments in 3 pairs arising from very short common basal stalk; dorsal and ventral pairs short-stalked, middle pair very short-stalked; dorsalmost and ventralmost filaments diverged from each other at angle of 90 degrees or a little less when viewed laterally; filaments decreasing in length and thickness to some extent from dorsal to ventral, dorsalmost longest filament $1.5-1.7 \mathrm{~mm}$ long, ventralmost shortest one 1.0-1.2 mm long; all filaments light yellow to light yellowish-brown, tapered 


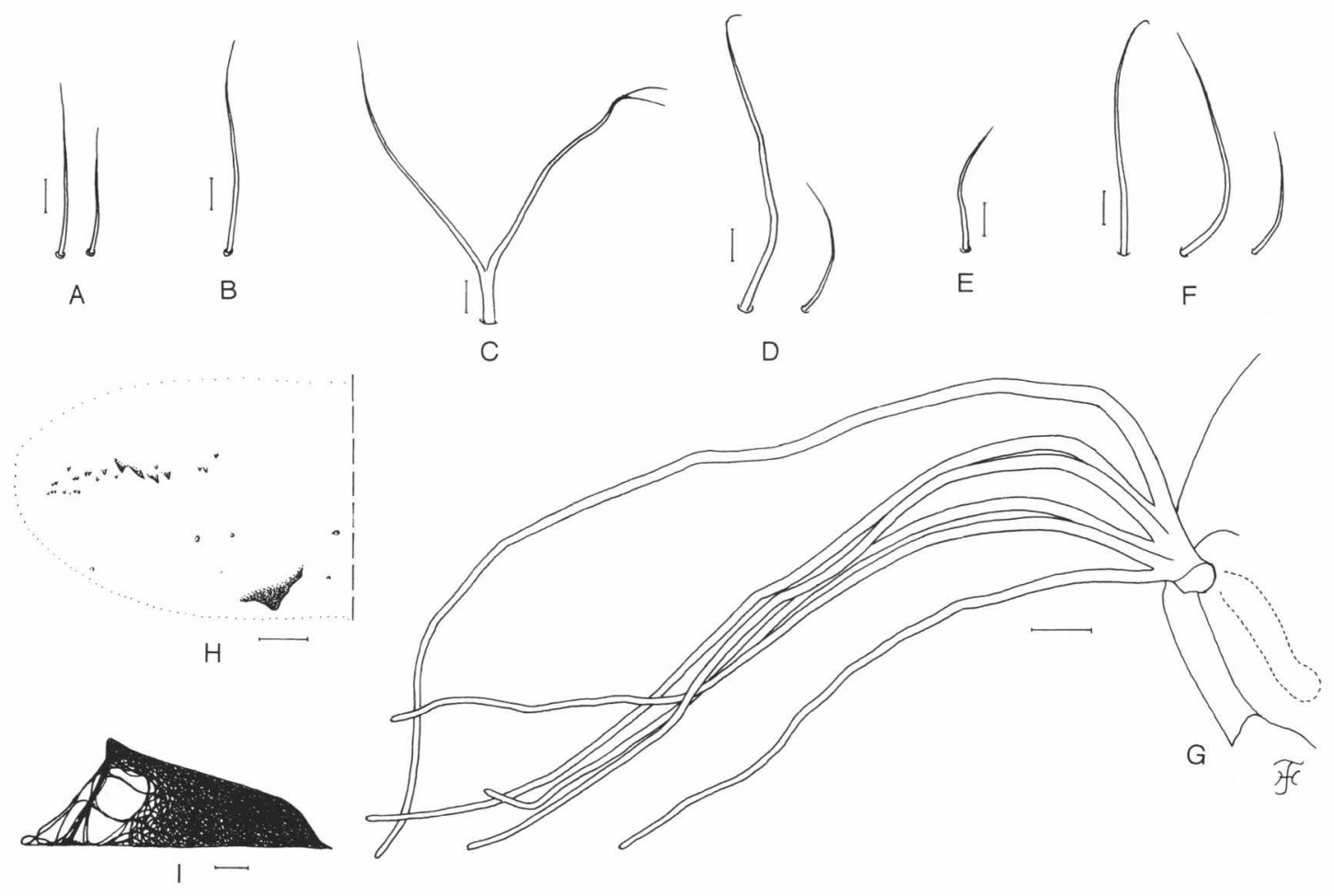

Fig. 3. Pupa of Simulium (Simulium) chungi sp. nov. A, frontal trichomes; B, facial trichome; C-F, trichomes on thorax (C, mediodorsal; D, anterolateral; E, posterolateral; F, ventrolateral); G, gill filaments (left side, outer view); H, dorsal surface of 9th abdominal segment showing terminal hook, comb-like groups of spines in transverse row (including 4 somewhat larger ones appearing spine-combs) and several small scattered tubercles (left half); I, cocoon (lateral view). Scale bars. 0.5 $\mathrm{mm}$ for $\mathrm{I} ; 0.1 \mathrm{~mm}$ for $\mathrm{G}$; $0.02 \mathrm{~mm}$ for $\mathrm{A}-\mathrm{F}$ and $\mathrm{H}$.

toward apex, with distinct annular ridges and furrows forming definite reticulate surface patterns, and densely covered with minute tubercles of different sizes (larger ones on ridges and smaller ones on interridge spaces). Abdomen. Dorsally, segment 1 weakly sclerotized, light yellowish-brown, with 1 medium-long simple slender seta on each side; segment 2 transparent, bare, with 1 medium-long simple slender seta and 5 short spinous setae on each side; segments 3 and 4 transparent, each with 4 distinct simple hooks and 1 short simple seta on each side; segments 5-9 transparent, with comb-like groups of minute spines on each side; segment 8 with distinct spine-combs in transverse row on each side, though segments 7 and 9 rarely with small spine-combs (Fig. $3 \mathrm{H}$ ); segment 9 with pair of cone-shaped terminal hooks and sparsely covered with several small tubercles (Fig. 3H). Ventrally, segments 3-9 transparent, each with comb-like groups of minute spines; segment 4 with a few slender minute setae on each side; segment 5 with pair of bifid stout hooks submedially and a few simple short setae on each side; segments 6 and 7 each with pair of bifid inner and simple outer stout hooks somewhat separated from each other, and a few simple short setae on each side. Grapnel-shaped hooklets absent on each side of segment 9. Cocoon (Fig. 3I). Wallpocket-shaped, thinly to moderately woven, with 1 or 2 large anterolateral windows (often together with a few to several smaller open spaces) on each side, not extending ventrolaterally; individual threads visible or invisible; light yellow to yellowish-brown; $4.0-5.0 \mathrm{~mm}$ long by $1.6-2.0 \mathrm{~mm}$ wide.

Mature larva. Body length $5.3-5.8 \mathrm{~mm}$. Body greyish-brown; abdomen gradually widened from segment 1 to segment 6 , then narrowed to posterior tip. Cephalic apotome (Fig. 4A) dark yellow to light brown except 


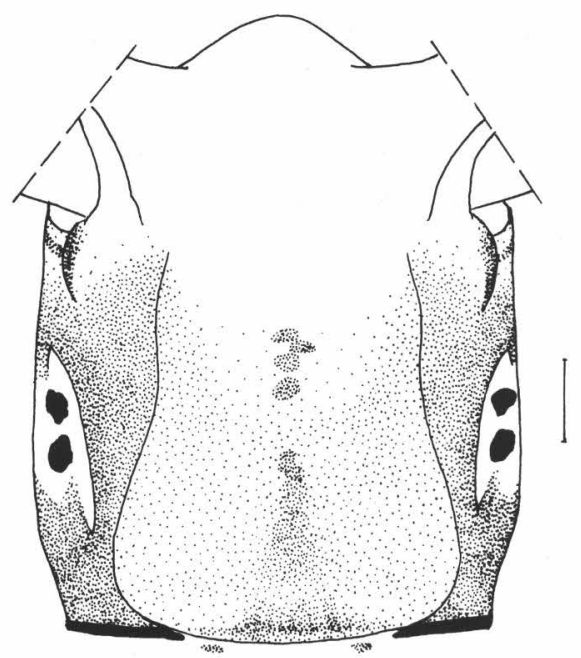

A

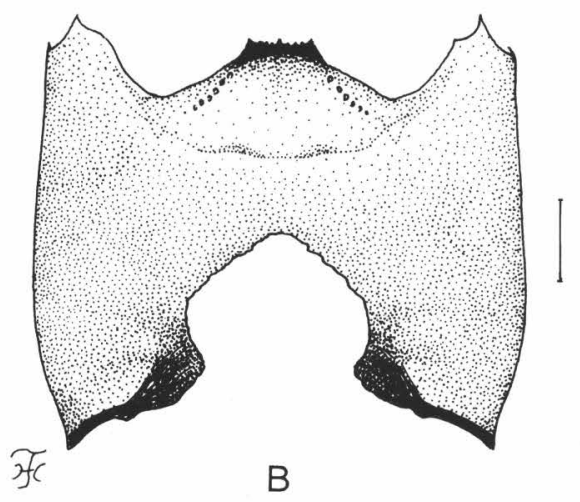

Fig. 4. Mature larva of Simulium (Simulium) chungi sp. nov. A, head capsule showing cephalic apotome and cervical sclerites (dorsal view); B, head capsule showing hypostoma and postgenal cleft (ventral view); C, apical portion of mandible; D, hypostoma. Scale bars. $0.1 \mathrm{~mm}$ for A and B; $0.02 \mathrm{~mm}$ for D; 0.01 $\mathrm{mm}$ for $\mathrm{C}$.

anterior $1 / 2$ usually pale yellow and with narrow portion along posterior margin light to dark brown; both anterior and posterior spots of median longitudinal spots medium to dark brown; mediolateral spots dark yellow to light brown (usually obscure or faintly positive); posterolateral spots yellow to dark yellow (usually obscure or appearing to be negative in some larvae). Lateral surface of head capsule entirely light to dark brown except eye-spot region yellow in some larvae, or light to dark brown except eye-spot region and lower $1 / 2$ and narrow area near anterior margin yellowish; eyebrow moderately to markedly defined; spots just before posterior margin light brown
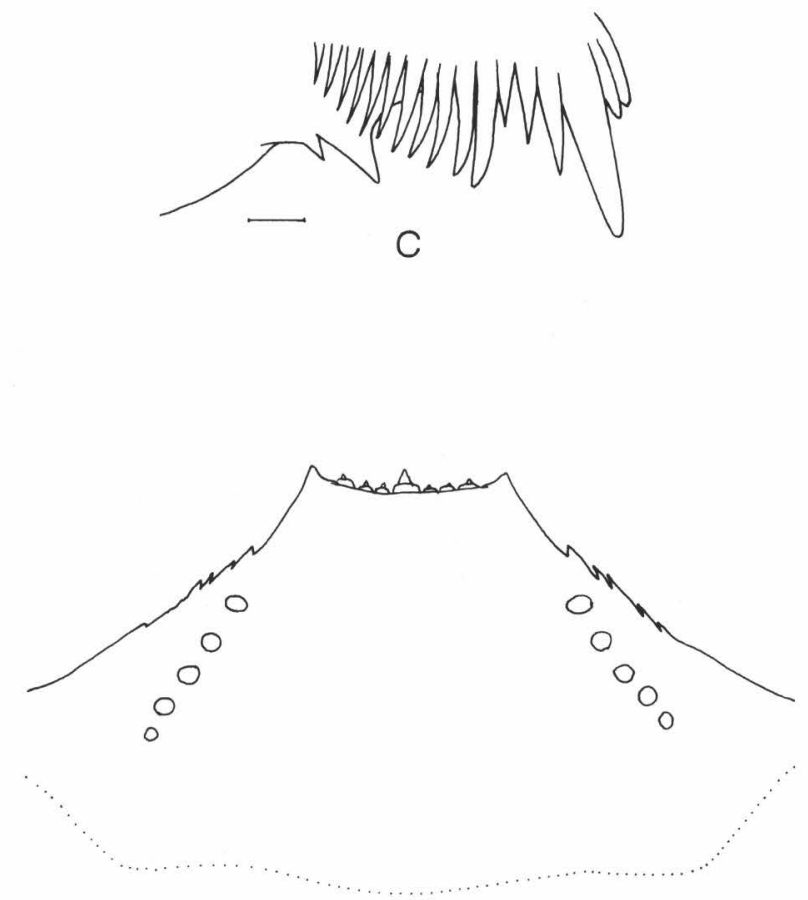

(usually faintly negative), isolated spot below eye-spot region light to medium brown (always positive). Ventral surface of head capsule (Fig. 4B) usually light to dark brown in most larvae but dark yellow in 1 larva, spot on each side of postgenal cleft light to dark brown (usually merged in background color). Antenna composed of 3 segments and apical sensillum, longer than stem of labral fan; proportional lengths of 1 st, 2 nd, and 3rd segments $1.0: 1.3$ : 0.7 . Labral fan with about 42 main rays. Mandible (Fig. 4C) with mandibular serrations composed of 1 large tooth and 1 small one; large tooth at obtuse angle with inner margin of mandible on apical side; supernumerary serra- 
tions absent; comb-teeth decreasing in length from 1 st tooth to 3rd one. Hypostoma (Fig. 4D) with 9 anterior teeth, of which median tooth and corner teeth are relatively larger than intermediate teeth; lateral margins moderately serrate apically; 5 or 6 hypostomal bristles diverged posteriorly from lateral border on each side. Postgenal cleft (Fig. 4B) deep, rounded, 2.1-2.5 times as long as postgenal bridge. Cervical sclerite composed of 1 small light brown elliptical piece (or plus 1 light brown rod-like piece) on each side. Thoracic cuticle almost bare. Abdominal cuticle bare except last segment moderately covered with short colorless setae on each side of anal sclerite. Rectal scales present. Rectal organ compound, with 10-15 finger-like secondary lobules per lobe. Anal sclerite X-shaped, with anterior arms 0.57 times as long as posterior ones; anterior arms broadened apically when viewed laterally; basal juncture area with deep narrow incision posteriorly; several sensilla present just posterior to posterior arms; accessory sclerite absent. Last abdominal segment lacking ventral papillae. Posterior circlet with 88-92 rows of hooklets with up to $15-17$ hooklets per row.

TYPE SPECIMENS. Holotype female with its associated pupal exuviae and cocoon, collected from Wushih River, crossing the national road No. 9 from Yilan to Shuanglienpi, Jhentou, Yilan county, Taiwan, 2. III. 2006, by H. Takaoka and Y. T. Huang. Paraty pes: 2 females, 2 males, all with their associated pupal exuviae and cocoon, and 6 mature larvae, same data and date as those of the holotype; 5 females and 3 males, all with their associated pupal exuviae and cocoon, Husi, near the entrance of Fushang Botanic Garden, Yilan county, 2. III. 2006, by H. Takaoka and Y. T. Huang.

ECOLOGICAL NOTES. The pupae and larvae of S. (S.) chungi sp. nov. were collected from fallen tree leaves in two moderately flowing mountainous streams: the first stream (width 8-10 m, depth $0.2-0.4$ $\mathrm{m}$, streambed with small to large stones, water temperature $14.3^{\circ} \mathrm{C}$, exposed to sun, altitude $50-60 \mathrm{~m}$ ) at Jhentou, and the second stream (width 2-4 m, depth 0.1-0.3 $\mathrm{m}$, streambed with small stones, water temperature $14.0^{\circ} \mathrm{C}$, partially shaded, altitude $600 \mathrm{~m}$ ) at Husi. Associated species were S. (S.) suzukii Rubtsov and S. (S.) sakishimaense Takaoka.

ETYMOLOGY. The specific name chung $i$ is in honor of Dr. Chung Chao-Lin, Center for Disease Control, Taiwan, Republic of China, for his great contribution to control of vector-borne diseases.

REMARKS. Simulium (S.) chungi sp. nov. is assigned to the griseifrons speciesgroup of the subgenus Simulium s. str., defined by Takaoka and Davies (1996), by having the haired basal section of the radial vein and the simple claw in the female, the style with long basal protuberance (Fig. 2D, F), the ventral plate nearly flat, with no distinct toothed posterior margin (Fig. 2G-I) in the male, and the gill with six filaments (Fig. 3G) in the pupa.

This new species is very similar to $S$. (S.) taipokauense Takaoka, Davies and Dudgeon described based on the males collected by a light-trap in Hong Kong (Takaoka et al., 1995) in many features including the numbers of vertical columns and horizontal rows of large upper-eye facets and the shape of the genitalia, but is barely distinguished by the following characteristics (those of S. (S.) taipokauense in parentheses): the relative length of the first flagellar segment of the antenna to the second one 1.5-1.6 (about 1.8), the sensory vesicle ellipsoidal or oblong and 0.24-0.28 times as long as the third segment (rounded and 0.18 times as long as the third segment), the mid trochanter medium brown except the basal $1 / 2$ yellow, the mid femur yellow to somewhat dark yellow except the apical cap light brown and the mid tibia yellow except the apical cap and the apical $1 / 2$ or more of the posterior surface slightly to moderately darkened (the trochanter, femur and tibia of the midleg all yellow).

Simulium (S.) chungi sp. nov. is also sim- 
ilar to the two Chinese species, $S$. (S.) $e p h$ ippioidum Chen and Wen described from Guizhou Province (Chen and Wen, 1999) and S. (S.) ledongense Yang and Chen described from Hainan Province (Yang and Chen, 2001), in that both species have the fully haired basal portion of the female radial vein, similar shape of the male genitalia, six pupal gill filaments and fenestrated cocoon. However, this new species is easily distinguished by the striated female scutum, the ovipositor valves having a narrow apical tip, the greater numbers of vertical columns and horizontal rows of the large upper-eye facets of the male, and the larval postgenal cleft rounded laterally.

\section{ACKNOWLEDGEMENTS}

We are grateful to Dr. C. L. Chung, Center for Disease Control, R.O.C., Taiwan, and Dr. H. Sasaki, Rakuno Gakuen University, Japan, who kindly supported and arranged our surveys in Taiwan. Thanks are due to Prof. K. J. Lin, National Chiayi University, Taiwan, for his kind help in providing useful information and items needed for the surveys, and also to Mr. W. L. Wu, Fo Guang College of Humanities and Social Sciences, for his kind cooperation in field surveys in Yilan county.

\section{REFERENCES}

Chen, H. B. and Wen, X. J. 1999. A new species of Simulium (Simulium) from Fanjing Mountain, Guizhou Province, China (Diptera: Simuliidae). Acta Zootaxonomica Sinica, 24: 436-439.

Chung, C. L. 1986. A new record of Simulium (Wallacellum) yonakuniense from Lanyu Is., Taitung county, Taiwan (Diptera: Simuliidae). J. Taiwan Mus., 39: 1-10.

Shiraki, T. 1935. Simuliidae of the Japanese Empire. Mem. Fac. Sci. Agric. Taihoku Imp. Univ., 16: 1-90.

Takaoka, H. 1979. The black flies of Taiwan (Diptera: Simuliidae). Pacific Insects, 20: 365-403.

Takaoka, H. 2003. The Black Flies (Diptera: Simuliidae) of Sulawesi, Maluku and Irian Jaya. xxii+581 pp., Kyushu University Press, Fukuoka.

Takaoka, H. and Davies, D. M. 1996. The Black Flies (Diptera: Simuliidae) of Java, Indonesia. viii +81 pp., Bishop Mus. Bull. Entomol. 6, Bishop Museum Press, Hawaii.

Takaoka, H., Davies, D. M. and Dudgeon, D. 1995. Black flies (Diptera: Simuliidae) from Hong Kong: Taxonomic notes with descriptions of two new species. Jpn. J. Trop. Med. Hyg., 23: 189-196.

Yang, M. and Chen, H. B. 2001. A new species of Simulium (Simulium) from Jianfeng Mountain, Hainan Province, China (Diptera: Simuliidae). Acta Zootaxonomica Sinica, 26: 90-93. 